\title{
MULTIPLICATIVE LINEAR FUNCTIONALS ON CONVOLUTION ALGEBRAS
}

\author{
PAUL AIZLEY
}

ABSTRACT. It is shown that semicharacters on the semigroup $S$ lead in a natural way to multiplicative linear functionals on $l(S)$, the convolution algebra of all complex valued functions on $S$. A theorem of D. H. Lehmer and a theorem of M. Tainiter follow as special cases.

1. Introduction. In [3] M. Tainiter proved and used a theorem with a long history. According to D. H. Lehmer [2] Daublebsky Von Sterneck stated and misproved a special case of the theorem in 1894. Lehmer generalized the theorem and correctly proved it in 1931 . Lehmer's theorem can be further extended and we do so below in Theorem 4.

\section{Notation.}

2.1. $S$ will always denote a commutative semigroup with an identity, $e$, (we could omit the identity but keep it for convenience). Also, for each $n \in S$ the set $\{(x, y) \in S \times S \mid x y=n\}$ is finite.

2.2. Let $I=\left\{x \in S \mid x^{2}=x\right\}$ be the set of idempotent elements in $S$.

2.3. Let $A_{n}=\{x \in S \mid x n=n\}$ be the set of associates of $n$.

2.4. Let $D_{n}=\{x \in S \mid \exists y \in S$ and $x y=n\}$ be the set of divisors of $n$. None of the sets $I, A_{n}$, and $D_{n}$ is empty.

2.5. Let $l(S)$ be the set of all complex-valued functions on $S$. Then $l(S)$ is a convolution algebra [4] with convolution given by

$$
f * g(n)=\sum_{x y=n} f(x) g(y) \text {. }
$$

\section{Semicharacters.}

3.1. Definition. Let $T$ be any semigroup. A semicharacter on $T$ is a bounded, multiplicative, complex-valued function $\chi$ on $T$ which is not identically zero. For our purposes we need another condition on $\chi$, i.e. $\{x \in T \mid \chi(x) \neq 0\}$ is finite. This is the only kind of semicharacter that we use, and we will refer to them simply as semicharacters.

4. THEOREM. If $\chi$ is a semicharacter on $S$, then the map $f \rightarrow$ $\sum_{x \in S} f(x) \chi(x)$ is a multiplicative linear functional on $l(S)$.

Received by the editors April 6, 1970.

AMS 1969 subject classifications. Primary 1005; Secondary 0510.

Key words and phrases. Semigroup, semicharacter, idempotents, associates, convolution algebra, multiplicative linear functional. 
Proof. Each sum below is finite so we rearrange the terms with impunity.

$$
\begin{aligned}
\left(\sum_{x \in S} f(x) \chi(x)\right)\left(\sum_{y \in S} g(y) \chi(y)\right) & =\sum_{x, y \in S} f(x) g(y) \chi(x y) \\
= & \sum_{n \in S}\left(\sum_{x y=n} f(x) g(y)\right) \chi(n)=\sum_{n \in S} f * g(n) \chi(n) .
\end{aligned}
$$

5. Lehmer's theorem. Let $N$ be the positive integers under any semigroup operation such that the conditions of $\$ 2$ are satisfied with $e=1$. In addition Lehmer postulates

$$
x y n \neq n \quad \text { implies } x n \neq n \text { and } y n \neq n \text {. }
$$

Equation (5.1) is enough to guarantee that $\chi_{n}$, the characteristic function of $A_{n}$ is a semicharacter on $N$.

For each $f \in l(N)$ define $F$ by

$$
F(n)=\sum_{x \in A_{n}} f(x)=\sum_{x \in N} f(x) \chi_{n}(x) .
$$

Then if $f * g=h$, we have by Theorem 4 that $H(n)=F(n) G(n)$ which is Theorem 4 of [2].

6. Tainiter's theorem. Let $T$ be a finite, commutative semigroup of idempotents. If $x \in D_{n}$, then for some $y \in T, x y=n$ and $x y n=n^{2}=n$, i.e. $x y \in A_{n}$. It follows that $x \in A_{n}$ since $x n=x(x y)=x^{2} y=x y=n$. So (5.1) holds and Theorem 4 in this case is Theorem 3.1 of [3].

For applications of Theorem 4 to combinatorial analysis see Tainiter [3]. For application to a class of convolution algebras and to elementary number theory see [1].

\section{REFERENCES}

1. P. Aizley, Structure theory for a class of convolution algebras, University Microfilms, Order \#69-16,475, Ann Arbor, Michigan.

2. D. H. Lehmer, On a theorem of von Sterneck, Bull. Amer. Math. Soc. 37 (1931), 723-726.

3. M. Tainiter, Generating functions on idempotent semigroups with application to combinatorial analysis, J. Combinatorial Theory 5 (1968), 273-288. MR 48 \#65.

4. E. Hewitt and H.S. Zuckerman, Finite dimensional convolution algebras, Acta. Math. 93 (1955), 67-119. MR 17, 1048.

University of Nevada, Las Vegas, Nevada 89109 